\title{
Towards a precise determination of the equation of state of QCD at high-temperature
}

\author{
Mattia Dalla Brida* \\ Dipartimento di Fisica, Università di Milano-Bicocca, and INFN, Sezione di Milano-Bicocca, \\ Piazza della Scienza 3, I-20126 Milano, Italy \\ E-mail: mattia.dallabrida@unimib.it

\section{Leonardo Giusti} \\ Dipartimento di Fisica, Università di Milano-Bicocca, and INFN, Sezione di Milano-Bicocca, \\ Piazza della Scienza 3, I-20126 Milano, Italy \\ E-mail: Leonardo.Giustidcern.ch

\section{Michele Pepe} \\ INFN, Sezione di Milano-Bicocca, Piazza della Scienza 3, I-20126 Milano, Italy \\ E-mail: Michele.Pepe@mib.infn.it
}

\begin{abstract}
We present preliminary results towards a fully non-perturbative determination of the equation of state of QCD at very high-temperatures, $T \approx 3-80 \mathrm{GeV}$. The key ingredient is the lattice formulation of QCD in a moving reference frame, which allows us for a neat determination of the entropy density from simple expectation values of the momentum-components of the energymomentum tensor. For the computation we employ $N_{\mathrm{f}}=3$ flavours of non-perturbatively $\mathrm{O}(a)$ improved Wilson-fermions. We present an analysis of the $\mathrm{O}(a)$-improvement of the expectation values entering the determination, and show how these can be accurately computed in simulations.
\end{abstract}

XIII Quark Confinement and the Hadron Spectrum - Confinement2018

31 July - 6 August 2018

Maynooth University, Ireland

${ }^{*}$ Speaker. 


\section{Introduction}

The equation of state $(\mathrm{EoS})$ is a fundamental quantity of QCD as it describes the thermodynamic properties of quarks and gluons at equilibrium. It is relevant for a wide spectrum of applications, including the physics of the early universe, the study of neutron stars, and the description of heavy-ion collisions. Tremendous progress has been made over the past decade in the determination of the EoS at zero chemical potential for temperatures $T \approx 500 \mathrm{MeV}$ and below, using lattice field theory methods [1, 2, 3]. Lattice QCD is indeed the only known framework that allows for a first-principles, non-perturbative computation of the EoS. On the other hand, all current state of the art determinations rely on (some variant of) the integral method [4]. This approach is very challenging from the computational point of view as one needs to accommodate within the same simulation the physics at zero-temperature and at the temperatures of interest. Due to this limitation, only very recently some first exploratory study touched temperatures $T \approx 1-2 \mathrm{GeV}$ [5].

In the past few years a new framework has been proposed for the determination of the EoS using lattice QCD. The idea is to consider QCD in a moving reference frame $[6,7,8] .{ }^{1}$ Within this framework one is able to completely decouple simulations at different temperatures, which makes computationally feasible to reach high-temperatures while having all uncertainties under control. The method has been very successfully applied to the SU(3) Yang-Mills theory [11, 12, 13, 14, 15]. The study of refs. $[12,14,15]$, in particular, obtained a determination of the EoS over two orders of magnitude in the temperature with half a per-cent accuracy. Following these encouraging results in the pure gauge sector, our goal is to obtain a similar determination in full QCD.

A first exploratory study in this direction was presented in [16], where some first numerical experience with the method was described. In this contribution we present some preliminary results towards a systematic determination of the $\mathrm{EoS}$ of $N_{\mathrm{f}}=3 \mathrm{QCD}$ in the completely unexplored regime of temperatures: $T \approx 3-80 \mathrm{GeV}$. This determination is of great interest for several reasons. First of all, it fills the gap in our knowledge of the EoS between temperatures of the order of the typical QCD scales, up to the electro-weak scale. This information is very relevant for understanding the physics of the early universe. Secondly, the results will provide an important, solid test of perturbation theory in the high-temperature regime. In this range of temperatures perturbation theory is commonly used to approximate the EoS. On the other hand, the results in the SU(3) Yang-Mills theory of $[12,14,15]$, clearly point to the fact that this might not be as accurate as it is typically assumed. It is thus compelling to assess this issue in the most relevant case of QCD. Finally, our computation intends to be the first determination of the EoS which systematically employs the Wilson rather than the staggered formulation of lattice QCD. Proving that precise results using Wilson-quarks are feasible with today's computational resources opens the way to further studies using this formulation. This is particularly relevant in view of consolidating the current state of the art determinations at lower temperatures.

The outline of this contribution is the following. In the next section we briefly review the framework of lattice QCD in a moving frame, and recall how the EoS can be computed in terms of simple expectation values of the energy-momentum tensor (EMT). In Sect. 3, we discuss our lattice set-up and give an analysis of the $\mathrm{O}(a)$-improvement of the relevant expectation values of EMT. In Sect. 4, we present the lines of constant physics we plan to follow for our high-temperature

\footnotetext{
${ }^{1}$ For other interesting approaches we recommend the reader to refs. [9, 10].
} 
determination of the EoS, and discuss some preliminary results for the bare expectation values of the EMT. We finally conclude with an outlook on future work.

\section{Thermodynamics from a moving frame}

Considering a thermal quantum field theory in a moving frame brings a new perspective in studying thermodynamics. This framework provides us indeed with new relations to compute thermodynamic potentials $[6,7,8]$. To define a thermal quantum field theory in a moving frame one must impose shifted boundary conditions (SBC) on the fields integrated over in the Euclidean path-integral. In the case of lattice QCD this amounts to requiring:

$$
U_{\mu}\left(L_{0}, \vec{x}\right)=U_{\mu}\left(0, \vec{x}-L_{0} \vec{\xi}\right), \quad \psi\left(L_{0}, \vec{x}\right)=-\psi\left(0, \vec{x}-L_{0} \vec{\xi}\right), \quad \bar{\psi}\left(L_{0}, \vec{x}\right)=-\bar{\psi}\left(0, \vec{x}-L_{0} \vec{\xi}\right) .
$$

In these equations, $L_{0}$ denotes the physical extent of the lattice in the temporal direction, while $\vec{\xi}$ is the so-called shift vector corresponding to the Euclidean velocity of the moving frame; note that in the rest frame, $\vec{\xi}=0$, the usual thermal/periodic boundary conditions are recovered. We then consider periodic boundary conditions for all fields in the three spatial dimensions of extent $L$.

The entropy density of the system, $s(T)$, is the central quantity of interest. Once $s(T)$ is known, the other thermodynamic potentials including the pressure $p(T)$ and the energy density $\varepsilon(T)$ can be inferred from standard thermodynamic identities. In a moving reference frame the entropy density is related to the momentum density of the system by [8]:

$$
\frac{s(T)}{T^{3}}=-\frac{L_{0}^{4}\left(1+\vec{\xi}^{2}\right)^{3}}{\xi_{k}}\left\langle T_{0 k}^{R}\right\rangle_{\xi}, \quad T^{-1}=L_{0} \sqrt{1+\vec{\xi}^{2}}
$$

where in this equation $\langle\cdot\rangle_{\xi}$ denotes the lattice path-integral expectation value in the presence of $\mathrm{SBC}$, and $T_{0 k}^{R}$ is the momentum $k$-component of the renormalized EMT. Eq. (2.2) is the natural transcription to a quantum field theory of the corresponding classical relativistic relation (cf. ref. [17]). In the following we discuss some of its relevant features when studied on the lattice.

\section{The energy-momentum tensor on the lattice}

\subsection{General considerations}

On the lattice the EMT requires renormalization due to the explicit breaking of Poincaré symmetry by the regularization. The problem of defining a properly renormalized EMT which respects, up to discretization errors, the correct Ward identities, was first addressed in a series of pioneering papers $[18,19,20]$. Focusing on the momentum-components of the EMT, the analysis of these references shows that properly renormalized fields can be defined as:

$$
T_{0 k}^{R}(x)=Z_{F}\left(g_{0}\right) T_{0 k}^{F}(x)+Z_{G}\left(g_{0}\right) T_{0 k}^{G}(x),
$$

where $T_{0 k}^{F}$ and $T_{0 k}^{G}$ are the bare fermionic and gluonic components of the EMT, respectively. The renormalization constants $Z_{F}$ and $Z_{G}$ appearing in this equation are renormalization scale independent, and thus only functions of the bare gauge coupling $g_{0}$. They depend on the specific 
discretization of the QCD action and of the bare fields $T_{0 k}^{F}$ and $T_{0 k}^{G}$, and they are fixed by imposing the validity of some continuum Ward identity at finite lattice spacing; only in this way we are guaranteed that in the continuum limit $T_{0 k}^{R}$ will converge to the generator of spatial translations. For our study we employ (non-perturbatively) $\mathrm{O}(a)$-improved Wilson-fermions and consider the Wilson gauge action [21]. For the bare EMT we take the discretized form given in eqs. (4) and (5) of ref. [16].

We have recently completed a 1-loop computation of the renormalization constants $Z_{F}$ and $Z_{G}$ within the framework of SBC. This calculation provides us with useful insight on how to choose practical renormalization conditions to fix $Z_{F}$ and $Z_{G}$ also non-perturbatively. In addition, the results give us valuable perturbative information on discretization effects: both to determine improvement coefficients (cf. Sect. 3.2), as well as to perturbatively subtract lattice artefacts from our non-perturbative determinations of the $Z$-factors. The details of this computation will be presented elsewhere [22]. Earlier computations of some of these renormalization factors using different setups can be found in refs. [18, 19, 20, 23, 24].

\section{2 $\mathrm{O}(a)$-improvement}

Wilson-fermions are known to be affected by discretization errors of $\mathrm{O}(a)$. If one aims at precise continuum extrapolations it is hence convenient to systematically remove them following Symanzik improvement programme [25, 26]. In short, this consists in adding irrelevant $\mathrm{O}(a)$ counterterms with the proper symmetry transformations to the lattice action and fields, and to tune their coefficients to cancel the unwanted $\mathrm{O}(a)$-effects in on-shell quantities. Given the fact that our action is $\mathrm{O}(a)$-improved, improved expectation values of the EMT are obtained by improving the EMT itself.

In this section we focus on the specific case of improving the expectation value $\left\langle T_{0 k}^{R}\right\rangle_{\xi}$; a more general discussion on the improvement of the EMT will be presented in [22]. We then begin by considering the situation where the quarks are massless. The $\mathrm{O}(a)$-counterterms we need to add to $T_{0 k}^{R}$ are given in principle by all mass dimension 5 operators which share the same lattice symmetries of these fields. Symmetry transformations and the field equations of motion can however be used to reduce the actual number of fields we need to consider in a specific (on-shell) expectation value [27]. Following the discussion of ref. [28], it is possible to show that an $\mathrm{O}(a)$-improved definition of $\left\langle T_{0 k}^{R}\right\rangle_{\xi}$ can be obtained as:

$$
\left\langle T_{I, 0 k}^{R}\right\rangle_{\xi}=Z_{G}\left(g_{0}\right)\left\langle T_{0 k}^{G}\right\rangle_{\xi}+Z_{F}\left(g_{0}\right)\left\{\left\langle T_{0 k}^{F}\right\rangle_{\xi}+a\left\langle\delta T_{0 k}^{F}\right\rangle_{\xi}\right\},
$$

where for the fields $\delta T_{0 k}^{F}$ we take:

$$
\delta T_{0 k}^{F}(x)=c_{T}^{F}\left(g_{0}\right) \frac{1}{8} \bar{\psi}(x)\left[\sigma_{0 \rho} \widehat{F}_{k \rho}(x)+\sigma_{k \rho} \widehat{F}_{0 \rho}(x)\right] \psi(x) .
$$

In this equation, $\widehat{F}_{\mu \nu}$ denotes the (traceless) clover discretization of the field strength tensor (see e.g. ref. [14] for its definition), while $c_{T}^{F}\left(g_{0}\right)$ is an improvement coefficient which must be tuned to remove the $\mathrm{O}(a)$-effects from eq. (3.2).

When considering the case of massive quarks more $\mathrm{O}(a)$-counterterms need to be added to the EMT. Here we discuss only the case of mass-degenerate quarks and leave the details of the non-degenerate case to [22]. We also assume that the bare gauge coupling $g_{0}$ is properly improved, 
and so the subtracted bare quark-masses, $m_{\mathrm{q}}=m_{0}-m_{\mathrm{cr}}\left(g_{0}\right)$, with $m_{0}$ the bare quark mass and $m_{\mathrm{cr}}$ its critical value (see ref. [27] for a discussion about this point). In this situation, an $\mathrm{O}(a)$-improved definition of $\left\langle T_{0 k}^{R}\right\rangle_{\xi}$ in the presence of mass-degenerate quarks is given by:

$$
\left\langle T_{I, 0 k}^{R}\right\rangle_{\xi}=Z_{G}\left(\widetilde{g}_{0}\right)\left\langle T_{I, 0 k}^{G}\right\rangle_{\xi}+Z_{F}\left(\widetilde{g}_{0}\right)\left\langle T_{I, 0 k}^{F}\right\rangle_{\xi}
$$

where $\widetilde{g}_{0}$ is the improved bare coupling [27], and

$$
T_{I, 0 k}^{G}(x)=\left(1+b_{T}^{G}\left(g_{0}\right) a m_{\mathrm{q}}\right) T_{0 k}^{G}(x), \quad T_{I, 0 k}^{F}(x)=\left(1+b_{T}^{F}\left(g_{0}\right) a m_{\mathrm{q}}\right)\left\{T_{0 k}^{F}(x)+a \delta T_{0 k}^{F}(x)\right\} .
$$

By a proper tuning of the $b$-coefficients, one can eliminate all $\mathrm{O}\left(a m_{\mathrm{q}}\right)$ discretization errors stemming from the EMT. It is easy to show that at tree-level in perturbation theory this is achieved by setting $b_{T}^{G}=0$ and $b_{T}^{F}=1[22]$.

The above discussion shows that, in general, in order to improve the expectation value $\left\langle T_{I, 0 k}^{R}\right\rangle_{\xi}$ we need to determine the improvement coefficients: $c_{T}^{F}, b_{T}^{F}$, and $b_{T}^{G}$. However, in the hightemperature regime of $N_{\mathrm{f}}=3 \mathrm{QCD}$, none of these coefficients is expected to be very relevant. At temperatures $T \gg 100 \mathrm{MeV}$, chiral symmetry is not broken for vanishing quark-masses; the only sources of chiral symmetry breaking at these temperatures are the quark-masses themselves. At high-temperature the chirally non-invariant $\mathrm{O}(a)$-counterterm (3.3) hence contributes to $\left\langle T_{I, 0 k}^{R}\right\rangle_{\xi}$ with $\mathrm{O}\left(a m_{\mathrm{q}}\right)$-effects; all discretization errors in $\left\langle T_{I, 0 k}^{R}\right\rangle_{\xi}$ are thus of $\mathrm{O}\left(a m_{\mathrm{q}}\right)$. Now, in order to keep discretization effects under control we must always be in the situation where $a T \ll 1$, which implies that: $a m_{\mathrm{q}} \ll m_{\mathrm{q}} / T$. Consequently, in $N_{\mathrm{f}}=3 \mathrm{QCD}$, where only the up, down, and strange quarks are considered, in the range of temperatures of interest, $T \approx 3-80 \mathrm{GeV}$, the $\mathrm{O}\left(a m_{\mathrm{q}}\right)$-effects affecting $\left\langle T_{I, 0 k}^{R}\right\rangle_{\xi}$ are expected to be well-below the per-cent level. In addition, small values of the lattice spacing, $a \ll T^{-1}$, correspond to relatively small values for the bare coupling $g_{0}$. Perturbative estimates for the improvement coefficients $c_{T}^{F}, b_{T}^{F}$, and $b_{T}^{G}$, are thus expected to be good enough to have these small $\mathrm{O}\left(a m_{\mathrm{q}}\right)$ effects under control. We plan to corroborate all these expectations by studying the size of the relevant $\mathrm{O}(a)$-counterterms non-perturbatively, so to be able to quantitatively estimate their effect in our results.

\section{Simulation strategy and results}

In this section we give some details on the lines of constant physics (LCPs) that we plan to follow for the determination of the entropy density in the high-temperature range, $T \approx 3-80 \mathrm{GeV}$. The aim is to obtain a final precision on the continuum entropy density of about $1 \%$ over the whole temperature range. The first step is to find values of the bare coupling $g_{0}$ and of the temporal resolution $L_{0} / a$ which correspond to a good set of constant physical temperatures to cover the desired range. To this end, we consider the LCPs defined by the ALPHA collaboration in terms of running couplings [29, 30, 31, 32, 33] (see ref. [34] for a recent review). Using in particular the results of refs. $[32,33]$ we are able to fix the bare parameters for 8 values of the temperature, where for each of these we can have up to 4 lattice spacings, corresponding to the resolutions: $L_{0} / a=6,8,10,12$. As for the choice of shift vector we take $\vec{\xi}=(1,0,0)$. Perturbative studies suggest that for these values of $L_{0} / a$, this should result in small discretization errors for the entropy (cf. ref. [16]). Given this choice and the results of ref. [31], we can infer from the values of the 
renormalized couplings used to fix the temperatures the physical value of the latter. Table 1 collects these results together with the bare parameters of the ensembles that we are currently generating. To conclude with the lattice geometry, we are considering for all ensembles a spatial resolution of $L / a=288$. This translates into having $T L \approx 34-17$ as we go from $L_{0} / a=6-12$. Since finite volume effects are exponentially small in $T L$ [8], we expect these effects to be well-below our target precision. We are currently conducting a systematic study to confirm that indeed finite volume effects in the relevant matrix elements of the EMT are negligible within our statistical precision.

\begin{tabular}{llllll}
\hline$T(\mathrm{GeV})$ & $L_{0} / a$ & $\beta$ & $\left\langle T_{0 k}^{G}\right\rangle_{\xi} / T^{4}$ & $\left\langle T_{0 k}^{F}\right\rangle_{\xi} / T^{4}$ & $N_{\mathrm{ms}}$ \\
\hline 2.8 & 6 & 6.2735 & $-2.361(19)$ & $-5.8667(94)$ & 100 \\
2.8 & 8 & 6.4680 & $-2.421(33)$ & $-5.700(18)$ & 250 \\
4.6 & 6 & 6.6050 & $-2.462(19)$ & $-5.9477(80)$ & 100 \\
7.5 & 6 & 6.9433 & $-2.562(18)$ & $-6.0265(95)$ & 100 \\
11.9 & 6 & 7.2618 & \multicolumn{2}{c}{ in production } & 20 \\
19.2 & 6 & 7.5909 & $-2.650(25)$ & $-6.1757(85)$ & 100 \\
30.5 & 6 & 7.9091 & \multicolumn{2}{c}{ in production } & 25 \\
47.8 & 6 & 8.2170 & $-2.707(18)$ & $-6.264(11)$ & 100 \\
76.5 & 6 & 8.5403 & $-2.806(23)$ & $-6.305(10)$ & 100 \\
76.5 & 8 & 8.7325 & $-2.784(40)$ & $-6.132(13)$ & 250 \\
\hline
\end{tabular}

Table 1: Values of the temperatures we are considering and corresponding values of $\beta=6 / g_{0}^{2}$ and $L_{0} / a$ of the ensembles that we are currently generating. All simulations have $L / a=288$. Our preliminary results for the bare gluonic and fermionic matrix elements of the EMT are also given, together with the total number of (independent) measurements we collected. We do not observe any autocorrelation between measurements on successive trajectories of length 2 MDUs.

For our choice of lattice action refs. [32, 33] give results for the critical value of the quarkmasses, $m_{\mathrm{cr}}\left(g_{0}\right)$. We employ these values and conveniently fix the bare quark masses $m_{0}=m_{\mathrm{cr}}\left(g_{0}\right)$ in our simulations. Up to discretization errors our 3-flavours of quarks can therefore be considered massless. Given the fact that physical quark-mass effects in the entropy are of $\mathrm{O}\left(m_{\mathrm{q}}^{2} / T^{2}\right)$, we expect that in our range of temperatures the difference with having physical values for the quark-masses is well-below our target accuracy. However, we plan to carefully study this issue by investigating the mass dependence of our results; this also in relation to estimate the size of the $\mathrm{O}\left(a m_{\mathrm{q}}\right)$ errors one would have for physical values of $m_{\mathrm{q}}$.

In table 1 we listed our preliminary results for the bare matrix elements, $\left\langle T_{0 k}^{G}\right\rangle_{\xi},\left\langle T_{0 k}^{F}\right\rangle_{\xi}$, for the ensembles we are currently considering. Given the large lattice volume we are simulating we obtain very precise results with only a few hundred measurements. At fixed $L_{0} / a$ and number of measurements the precision we reach does not seem to depend too strongly on the temperature. For $L_{0} / a=6$, we typically reach a precision on the gluonic matrix element of $\approx 0.6-0.9 \%$, while for the fermionic contribution we have $\approx 0.15 \%$ accuracy at all temperatures. When changing $L_{0} / a$ at fixed $\beta$, we (roughly) observe the expected scaling of the relative error with $\left(L_{0} / a\right)^{4}$. Finally, we may gain some rough idea on cutoff effects in the entropy density at the highest temperatures by taking for the renormalization factors $Z_{F}, Z_{G}$ their 1-loop values [22]. If we consider in particular the ratio between the entropy density obtained for $L_{0} / a=6$ and 8 at $T_{\mathrm{pt}}=76.5 \mathrm{GeV}$, we find: 
$\left.\left[s\left(T_{\mathrm{pt}}, L_{0} / a=8\right) / s\left(T_{\mathrm{pt}}, L_{0} / a=6\right)\right]\right|_{Z_{F, G}^{1-\text { loop }}} \approx 0.98$, which means that discretization errors are of the order of $2 \%$. Bearing in mind that the non-perturbative $Z$-factors will also carry their cutoff effects and statistical errors, safe continuum limit extrapolations with $1 \%$ accuracy seem at hand.

\section{Conclusions and outlook}

In this contribution we presented some preliminary results towards a per-cent accuracy determination of the EoS of $N_{\mathrm{f}}=3 \mathrm{QCD}$ in the range of temperatures: $T \approx 3-80 \mathrm{GeV}$. The results we obtain for the bare matrix elements of the EMT are very encouraging: we can reach very highprecision with modest computational resources. In addition, the set-up allows us to have systematic effects under control, including both finite-volume and discretization effects. In order to better understand the nature and size of the latter we have studied in some detail the $\mathrm{O}(a)$-improvement of the relevant expectation values of the EMT. The next mandatory step at this point is the determination of the renormalization constants $Z_{F}, Z_{G}$. We already devised a set of non-perturbative renormalization conditions and tested their viability to 1-loop order in lattice perturbation theory [22]. Their non-perturbative determination is on going.

\section{Acknowledgements}

The code used for the simulations is based on the openQCD-1 . 6 package $[35,36]$. All simulations were performed on the HPC cluster Wilson at the University of Milano-Bicocca, and on the Marconi machine at CINECA, through agreements of INFN and the University of Milano-Bicocca with CINECA. We gratefully acknowledge the computer resources and the technical support provided by these institutions.

\section{References}

[1] S. Borsanyi et al., Full result for the QCD equation of state with 2+1 flavors, Phys. Lett. $\mathbf{B 7 3 0}$ (2014) 99-104.

[2] G. S. Bali, F. Bruckmann, G. Endrödi, S. D. Katz, and A. Schäfer, The QCD equation of state in background magnetic fields, JHEP 08 (2014) 177.

[3] HotQCD Collaboration, A. Bazavov et al., Equation of state in ( $2+1$ )-flavor QCD, Phys. Rev. D90 (2014) 094503.

[4] G. Boyd et al., Thermodynamics of SU(3) lattice gauge theory, Nucl. Phys. B469 (1996) 419-444.

[5] A. Bazavov, P. Petreczky, and J. H. Weber, Equation of State in 2+1 Flavor QCD at High Temperatures, Phys. Rev. D97 (2018), no. 1014510.

[6] L. Giusti and H. B. Meyer, Thermal momentum distribution from path integrals with shifted boundary conditions, Phys. Rev. Lett. 106 (2011) 131601.

[7] L. Giusti and H. B. Meyer, Thermodynamic potentials from shifted boundary conditions: the scalar-field theory case, JHEP 11 (2011) 087.

[8] L. Giusti and H. B. Meyer, Implications of Poincare symmetry for thermal field theories in finite-volume, JHEP 01 (2013) 140. 
[9] WHOT-QCD Collaboration, K. Kanaya, S. Ejiri, R. Iwami, M. Kitazawa, H. Suzuki, Y. Taniguchi, and T. Umeda, Equation of state in $(2+1)$-flavor QCD at physical point with improved Wilson fermion action using gradient flow, EPJ Web Conf. 175 (2018) 07023.

[10] M. Caselle, A. Nada, and M. Panero, QCD thermodynamics from lattice calculations with nonequilibrium methods: The SU(3) equation of state, Phys. Rev. D98 (2018), no. 5054513.

[11] D. Robaina and H. B. Meyer, Renormalization of the momentum density on the lattice using shifted boundary conditions, PoS LATTICE2013 (2014) 323.

[12] L. Giusti and M. Pepe, Equation of state of a relativistic theory from a moving frame, Phys. Rev. Lett. 113 (2014) 031601.

[13] T. Umeda, Fixed-scale approach to finite-temperature lattice QCD with shifted boundaries, Phys. Rev. D90 (2014), no. 5054511.

[14] L. Giusti and M. Pepe, Energy-momentum tensor on the lattice: Nonperturbative renormalization in Yang-Mills theory, Phys. Rev. D91 (2015) 114504.

[15] L. Giusti and M. Pepe, Equation of state of the SU(3) Yang-Mills theory: A precise determination from a moving frame, Phys. Lett. $\mathbf{B 7 6 9}$ (2017) 385-390.

[16] M. Dalla Brida, L. Giusti, and M. Pepe, QCD in a moving frame: an exploratory study, EPJ Web Conf. 175 (2018) 14012.

[17] L. D. Landau and E. M. Lifschits, Fluid Mechanics, vol. 6 of Course of Theoretical Physics. Pergamon Press, Oxford, 1975.

[18] S. Caracciolo, G. Curci, P. Menotti, and A. Pelissetto, The Energy Momentum Tensor for Lattice Gauge Theories, Annals Phys. 197 (1990) 119.

[19] S. Caracciolo, P. Menotti, and A. Pelissetto, Analytic determination at one loop of the energy momentum tensor for lattice QCD, Phys. Lett. B260 (1991) 401-406.

[20] S. Caracciolo, P. Menotti, and A. Pelissetto, One loop analytic computation of the energy momentum tensor for lattice gauge theories, Nucl. Phys. B375 (1992) 195-239.

[21] JLQCD, CP-PACS Collaboration, N. Yamada et al., Non-perturbative O(a)-improvement of Wilson quark action in three-flavor QCD with plaquette gauge action, Phys. Rev. D71 (2005) 054505.

[22] M. Dalla Brida, L. Giusti, and M. Pepe, in preparation.

[23] S. Capitani and G. Rossi, Deep inelastic scattering in improved lattice QCD. 1. The First moment of structure functions, Nucl. Phys. B433 (1995) 351-389.

[24] G. Burgio, S. Caracciolo, and A. Pelissetto, Algebraic algorithm for the computation of one loop Feynman diagrams in lattice QCD with Wilson fermions, Nucl. Phys. B478 (1996) 687-722.

[25] K. Symanzik, Continuum Limit and Improved Action in Lattice Theories. 1. Principles and $\phi^{4}$ Theory, Nucl. Phys. B226 (1983) 187-204.

[26] K. Symanzik, Continuum Limit and Improved Action in Lattice Theories. 2. O(N) Nonlinear Sigma Model in Perturbation Theory, Nucl. Phys. B226 (1983) 205-227.

[27] M. Lüscher, S. Sint, R. Sommer, and P. Weisz, Chiral symmetry and $O($ a) improvement in lattice QCD, Nucl. Phys. B478 (1996) 365-400.

[28] S. Capitani, M. Göckeler, R. Horsley, H. Perlt, P. E. L. Rakow, G. Schierholz, and A. Schiller, Renormalization and off-shell improvement in lattice perturbation theory, Nucl. Phys. $\mathbf{B 5 9 3}$ (2001) 183-228. 
[29] ALPHA Collaboration, M. Dalla Brida et al., Determination of the QCD $\Lambda$-parameter and the accuracy of perturbation theory at high energies, Phys. Rev. Lett. 117 (2016), no. 18182001.

[30] ALPHA Collaboration, M. Dalla Brida et al., Slow running of the Gradient Flow coupling from 200 MeV to 4 GeV in $N_{\mathrm{f}}=3$ QCD, Phys. Rev. $\mathbf{D 9 5}$ (2017), no. 1014507.

[31] ALPHA Collaboration, M. Bruno et al., QCD Coupling from a Nonperturbative Determination of the Three-Flavor $\Lambda$ Parameter, Phys. Rev. Lett. 119 (2017), no. 10102001.

[32] ALPHA Collaboration, M. Dalla Brida, P. Fritzsch, T. Korzec, A. Ramos, S. Sint, and R. Sommer, $A$ non-perturbative exploration of the high energy regime in $N_{\mathrm{f}}=3$ QCD, Eur. Phys. J. C78 (2018), no. 5372 .

[33] ALPHA Collaboration, I. Campos, P. Fritzsch, C. Pena, D. Preti, A. Ramos, and A. Vladikas, Non-perturbative quark mass renormalisation and running in $N_{f}=3$ QCD, Eur. Phys. J. C78 (2018), no. 5387.

[34] ALPHA Collaboration, M. Dalla Brida, Precision Determination of $\alpha_{s}$ from Lattice QCD, Universe 4 (2018), no. 12148.

[35] M. Lüscher and S. Schaefer, Lattice QCD with open boundary conditions and twisted-mass reweighting, Comput. Phys. Commun. 184 (2013) 519-528.

[36] openQCD package, Simulation program for lattice $Q C D$, http: // luscher.web. cern. ch/luscher/openQCD/. 